\title{
Mixed state microwave resistivity of cuprate superconductors
}

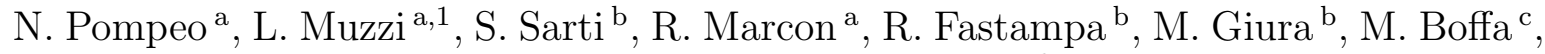 \\ M.C. Cucolo ${ }^{\mathrm{c}}$, A.M. Cucolo ${ }^{\mathrm{c}}$, C. Camerlingo ${ }^{\mathrm{d}}$, and E. Silva ${ }^{\mathrm{a}}$. \\ a Dipartimento di Fisica "E. Amaldi" and INFM, Università di Roma Tre, Via della Vasca Navale 84, I-00146 Roma, Italy. \\ ${ }^{\mathrm{b}}$ Dipartimento di Fisica and INFM, Università di Roma "La Sapienza", I-00185 Roma, Italy. \\ ${ }^{\mathrm{c}}$ Dipartimento di Fisica and INFM, Università di Salerno, Baronissi, Salerno, Italy. \\ ${ }^{\mathrm{d}}$ CNR - Istituto di Cibernetica "E. Caianiello", Napoli, Italy.
}

\begin{abstract}
We present a compared experimental investigation of the $(a, b)$ plane vortex-state complex resistivity at $48 \mathrm{GHz}$ in $\mathrm{YBa}_{2} \mathrm{Cu}_{3} \mathrm{O}_{7-\delta}, \mathrm{SmBa}_{2} \mathrm{Cu}_{3} \mathrm{O}_{7-\delta}$ and $\mathrm{Bi}_{2} \mathrm{Sr}_{2} \mathrm{CaCu}_{2} \mathrm{O}_{8+x}$. In $\mathrm{YBa}_{2} \mathrm{Cu}_{3} \mathrm{O}_{7-\delta}$ and $\mathrm{SmBa}_{2} \mathrm{Cu}_{3} \mathrm{O}_{7-\delta}$ the field dependence of the response can be consistently described by a combination of flux flow and strong pair breaking due to the presence of lines of nodes in the gap. In $\mathrm{Bi}_{2} \mathrm{Sr}_{2} \mathrm{CaCu}_{2} \mathrm{O}_{8+x}$, by contrast, the data might be described by the pair breaking alone.
\end{abstract}

Key words: A.superconductors, A.thin films, D.electrical conductivity

PACS: $74.78 . \mathrm{Bz}, 74.25 . \mathrm{Op}$

\section{Introduction}

In high- $T_{c}$ cuprate superconductors (HTCS) the microwave response has been extensively investigated in order to get information, among the others, on the symmetry of the order parameter, on the vortex parameters such as the vortex viscosity and pinning frequency $[1,2]$ and on the temperature dependence of the superfluid fraction (via the measurement of the temperature dependence of the London penetration depth [3]). In the vortex state the response has been analysed mainly in terms of vortex motion, often assuming that the field dependence of the quasiparticle (QP) and

\footnotetext{
Email address: pompeo@fis.uniroma3.it (N. Pompeo).

1 Present address: ENEA, Frascati, Italy.
}

the superfluid (SF) could be neglected. However, there are some experimental results that point to a very relevant role of $\mathrm{QP} / \mathrm{SF}$ in the determination of the high-frequency response in the vortex state. In particular, in $\mathrm{Bi}_{2} \mathrm{Sr}_{2} \mathrm{CaCu}_{2} \mathrm{O}_{8+x}$ (BSCCO) it has been shown that, at low enough temperatures, the imaginary sub-terahertz conductivity is entirely due to the strong field dependent superfluid depletion in a superconductor with lines of nodes in the gap [4]. Moreover, we have recently shown [5] that in $\mathrm{SmBa}_{2} \mathrm{Cu}_{3} \mathrm{O}_{7-\delta}(\mathrm{SmBCO})$ thin films the $48 \mathrm{GHz}$ microwave resistivity is made up by two markedly different contributions, namely a linear (in the field induction $B$ ) term, due to the vortex motion, that appears in the real part of the microwave resistivity only (due to the fact that at high frequencies the vortex motion is purely dissi- 
pative), and a sublinear one, $\sim \sqrt{B}$, which reflects the strong pair breaking outside the vortex cores [6]. Noticeably, in SmBCO the field-dependent $\mathrm{QP} / \mathrm{SF}$ response is detected up to very close to $T_{c}$. Aim of this paper is to present a compared study of the microwave response of $\mathrm{SmBCO}$, $\mathrm{YBa}_{2} \mathrm{Cu}_{3} \mathrm{O}_{7-\delta}(\mathrm{YBCO})$ and $\mathrm{BSCCO}$ in the vortex state in moderate fields, for temperatures not too far from $T_{c}$. By assuming that the linear term in the real resistivity is due to vortex motion, we can directly extract the complex QP/SF conductivity. We find that YBCO and SmBCO behave similarly, and that the description based on flux flow $+\mathrm{SF}$ depletion is fully consistent with the data. By contrast, this framework does not apply to BSCCO, where it seems that vortex motion does not play any relevant role in our temperature and field ranges. In this compound, the conductivity seems to be due solely to field induced $\mathrm{QP} / \mathrm{SF}$ variations.

\section{Experimental}

The samples under investigation are highly oriented, $200 \mathrm{~nm}$ thin films of YBCO, SmBCO and BSCCO. Preparation details and crystallographic characterization are reported elsewhere $[7,8]$. Critical temperatures are $87,86.5$ and 89 $\mathrm{K}$ respectively, as estimated from the crossing of the microwave real and imaginary fluctuation conductivity [9] (the typical $\pm 0.5 \mathrm{~K}$ uncertainty of this method is inessential for the purposes of the present paper). The $(a, b)$-plane complex resistivity $\tilde{\rho}$ is measured with a metal cylindrical resonant cavity, operating in the $\mathrm{TE}_{011}$ mode at $\nu_{0}=48.2 \mathrm{GHz}$ in the end-wall-replacement configuration. A moderate magnetic field, aligned with the $\mathrm{c}$ axis, is swept at each temperature from 0 to $B \simeq \mu_{0} H=0.8 \mathrm{~T}$ (we expect that the approximate equality between applied and internal field can be inaccurate at very low fields only). In the temperature range here explored, $T>70 \mathrm{~K}$, the thin-film approximation is justified [10] and measurements of the field-induced shifts of $Q$ factor and $\nu_{0}$ yield the field-induced variations of the complex resistivity, $\Delta \tilde{\rho}(B)$, at fixed temperatures. The deter- mination of $\Delta \tilde{\rho}(B)$ requires only the knowledge of geometrical factors; absolute values of $\tilde{\rho}$ require the cavity calibration. In the following we will use normalized resistivities, defined as $\tilde{r}=r_{1}+\mathrm{i} r_{2}=$ $\frac{\tilde{\rho}}{\rho_{0}}$, with $\rho_{0}=\rho_{1}(100 K)$, and the corresponding normalized field variations $\Delta \tilde{r}=\tilde{r}(B)-\tilde{r}(0)$. Experimental results are as follow: the two REBCO samples show a similar behaviour in the entire temperature range examined up to near $T_{c}$. Measurements, plotted as a function of $\sqrt{B}$, are reported in fig. 1 for a selected set of field sweeps at different temperatures for the YBCO sample.

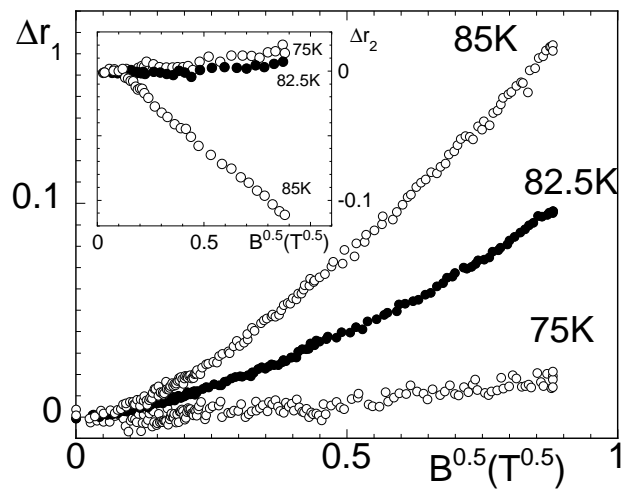

Fig. 1. Normalized complex resistivity vs $\sqrt{B}$ in YBCO at selected temperatures. Main panel: $\Delta r_{1}$; inset: $\Delta r_{2}$.

It can be noted that $\Delta r_{2}$ is well approximated by a straight line, which corresponds to a $\sim \sqrt{B}$ dependence. Moreover, $\Delta r_{2}$ changes from positive to negative as the temperature increases, but without changing the functional dependence on the magnetic field. On the other hand, $\Delta r_{1}$ is always positive: an additional upward curvature in the data plotted as a function of $\sqrt{B}$ indicates the presence of both a square root and a linear term in the $B$ dependence.

We now examine the measurements in BSCCO, where significant differences with respect to the behaviour of REBCO are observed (fig. 2). In the temperature region above $78 \mathrm{~K} \Delta r_{2}(B)$ is always decreasing, but shows a $B$ dependence made up of a combination of a linear term and a square-root one. $\Delta r_{1}(B)$, on the other hand, has essentially a $\sqrt{B}$ dependence up to $85 \mathrm{~K}$, where it starts to change to a linear $B$ dependence, which holds up to $95 \mathrm{~K}$ (well above $T_{c}$ ). It appears that the behaviour in BSCCO 


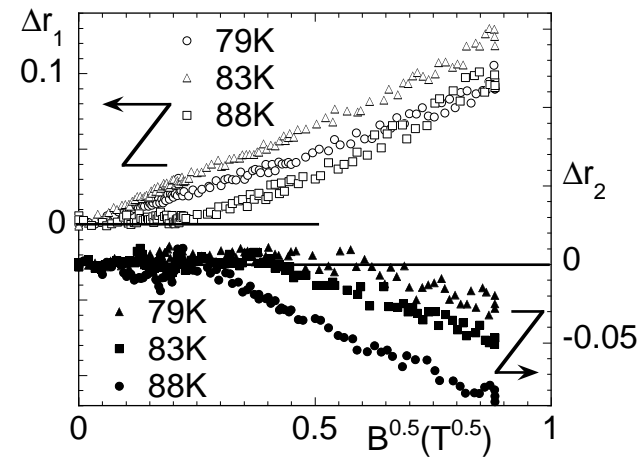

Fig. 2. Normalized complex resistivity vs $\sqrt{B}$ in BSCCO at selected temperatures. Open symbols: $\Delta r_{1}$; full symbols: $\Delta r_{2}$.

is somewhat reversed with respect to $\mathrm{SmBCO}$ and YBCO, exhibiting a dominant $\sqrt{B}$ term in the real part of the resistivity and a significant $\sim B$ term in the imaginary part. We will discuss in the following section the indications that can be gained from this experimental behaviour.

\section{Discussion}

As extensively discussed in [5], at high enough frequencies, low fields, and not too close to $T_{c}$, the vortex motion and QP/SF contributions to the microwave complex resistivity are additive, and one can write $\tilde{r}(B, T)=\tilde{r}(0, T)+\Delta \tilde{r}(B, T)$, with:

$\Delta r_{1}=\Delta r_{1}^{v m}+\Delta r_{1}^{q p s f} \simeq b_{1}(T) B+a_{1}(T) \sqrt{B}$
$\Delta r_{2}=\Delta r_{2}^{v m}+\Delta r_{2}^{q p s f} \simeq b_{2}(T) B+a_{2}(T) \sqrt{B}$

where we have explicitly indicated the vortex motion and the $\mathrm{QP} / \mathrm{SF}$ terms, the latter approximate equality holds as an expansion for small fields, and we have cosidered the fact that the superfluid fraction decreases as $\sqrt{B}$ in a superconductor with lines of nodes [6]. In this framework, $b_{1,2}$ are related to vortex motion. We note that the knowledge of $\tilde{r}(0, T)$ and of the vortex motion terms $b_{1,2}$ allows for the inversion of the $\mathrm{QP} / \mathrm{SF}$ terms and the determination of the field-dependent conductivity due to to QP and SF only.

We will discuss the data in the light of Eq.s 1,2. Due to their similarity, we discuss first the results in YBCO and SmBCO, and we successively consider the results in BSCCO. The experimental results obtained for the two REBCO samples find a very satisfactory explanation in terms of Eq.s 1,2 in the free-flux-flow limit (see [5] for an extended data presentation and discussion in SmBCO). Therefore, once the coefficient $b_{1,2}(T)$ are known, the $\mathrm{QP} / \mathrm{SF}$ conductivity can be extracted by subtracting the linear terms from the total resistivity and inverting the relation. The result is given in fig. 3 for the superfluid (imaginary) conductivity $\sigma_{2}$ in SmBCO and YBCO: it can be clearly seen that $\sigma_{2}$ shows a remarkable $\sqrt{B}$ trend, in agreement with the prediction of the $\sqrt{B}$ dependence for the superfluid density in a nodal superconductor.

For the BSCCO sample we initially note that above $\sim 88 \mathrm{~K} \Delta r_{1}$ has evolved from a square root to a pure linear $B$ dependence. Since the latter field dependence continues through and above the critical temperature, we argue that at high temperatures the response is dominated by fluctuations, which we will analyze in a future work. We therefore consider the temperature range below $88 \mathrm{~K}$. We first note that the imaginary resistivity is a decreasing function of the magnetc field, thus ruling out possible strong pinning effects (we remind that conventional vortex motion requires $\Delta r_{2}>0$ in the strong pinning regime $[1,11])$. It would be then natural to apply the framewok based on flux flow + pair breaking, as successfully applied in REBCO. However, this approach does not appear to be consistent: in fact, there is no evident linear term in the real part of the field-induced resistivity variation, while a linear term is present in the imaginary part. This is somewhat puzzling in the frame of a conventional vortex dynamics. While complex vortex dynamics cannot be definitely ruled out, in order to explore other alternative scenarios we consider a somewhat opposite (and oversimplified) view: we assume that the measured complex resistivity is entirely determined by the field dependence of charge carriers conductivity, with no relevant contribution from the vortex motion. In this frame, the measured $\tilde{\rho}(B)$ can be directly inverted to obtain $\sigma_{2}$. The result is given in fig. 3, lower panel, in which $\sigma_{2}$ shows a remarkable $\sqrt{B}$ trend up to near $T_{c}$, where it deviates upon entering the different (fluctuational) regime. The total irrelevance of 


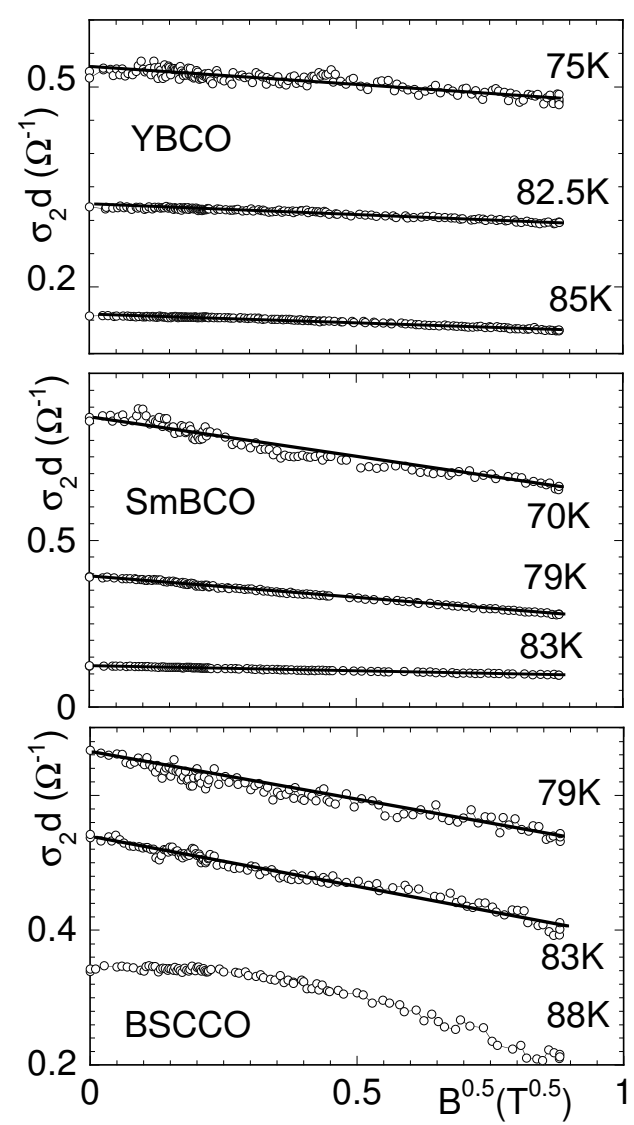

Fig. 3. Superfluid conductance ( $d$ is the film thickness) vs $\sqrt{B}$ at selected temperatures in $\mathrm{YBCO}, \mathrm{SmBCO}$ and BSCCO (from top to bottom). The $\sim \sqrt{B}$ behaviour, as expected for a superconductor with lines of nodes in the gap is ubiquitous (straight lines through the data points are guides for the eye). Only in BSCCO at high temperatures the field dependence switches to linear, $\sim B$.

vortex motion contribution is clearly an oversimplification, and more accurate models should take the vortex motion into account. Nevertheless, neglecting the vortex contribution brings the $\mathrm{QP} / \mathrm{SF}$ conductivity of BSCCO in close similarity to the one obtained in YBCO and SmBCO.

\section{Conclusions}

We have reported measurements of the complex resistivity $\tilde{\rho}$ in a static applied field for three HTS materials, YBCO, SmBCO and BSCCO. Both RE-
BCO samples showed a $B$ dependence satisfactorily explained as standard vortex dynamics added to a $\sqrt{B}$ pair-breaking term for the superfluid density, as expected for superconductors with nodes in gap. On the other hand, the BSCCO sample showed a field-induced variation of $\tilde{\rho}$ inconsistent with standard vortex dynamics. By contrast, exploiting an oversimplified model where no relevant vortex contribution is present, and the whole response is due to the quasiparticle increase due to the strong field-induced pair breaking in a nodal supeconductor, the obtained superfluid conductivity $\sigma_{2}$ is found to be consistent with theoretical expectations and with the $\sigma_{2}$ determined in REBCO. In all cases it appears that field induced QP excitations play an essential role, as witnessed by the clear $\sqrt{B}$ dependence of $\sigma_{2}$.

Acknowledgements

This work has been partially supported by Italian MIUR under FIRB project "Strutture semiconduttore/superconduttore per l'elettronica integrata".

\section{References}

[1] M. Golosovsky, M. Tsindlekht, D. Davidov, Supercond. Sci. Technol. 9 (1996) 1

[2] Y. Tsuchiya et al., Phys. Rev. B 63 (2001) 184517.

[3] W.N. Hardy et al.Phys. Rev. Lett. 70, 3999 (1993).

[4] R. Mallozzi et al.Phys. Rev. Lett. 81, 1485 (1998).

[5] E. Silva et al., cond-mat/0405324

[6] G.E. Volovik, JETP Lett. 58 (1993) 469; H. Won and K. Maki, Phys. Rev. B 53 (1996) 5927.

[7] M.A. Boffa et al., Physica C 384, 419 (2003).

[8] C. Camerlingo et al.presented at INFMeeting, June 2002, Bari, Italy (unpublished)

[9] E. Silva et al., Eur. Phys. J. B 37 (2004) 277

[10] E. Silva et al., Supercond. Sci. Technol. 9 (1996) 934.

[11] M.W. Coffey and J.R.Clem, Phys. Rev. Lett. 67 (1991) 386. 